\title{
Forecasting the next handoff for users moving with the Random Waypoint mobility model
}

\author{
Enrica Zola*, Francisco Barcelo-Arroyo and Israel Martín-Escalona
}

\begin{abstract}
Users in a cellular network can move while their connections are handed off to different access points. Studies prove that the mobility pattern followed have a strong impact on performance metrics (i.e., handoff $(\mathrm{HO})$ rate, cell residence time). Recently, some key aspects of the Random Waypoint mobility model have been studied in depth, but relating those studies with different cellular layouts has not been reported. Interest in forecasting the cell to which a device may be handed off depending on the movement pattern is twofold. First, it gives insight into properties and statistics of the mobility model. Second, and from a more practical perspective, it is useful to manage resource allocation and reservation strategies in order to smooth the $\mathrm{HO}$ process. The goal of this article is to provide an analytical framework for these predictions in a simple layout. Given a node's current location and the timestamp and location of the last waypoint, an approximation for $\mathrm{HO}$ during time $\Delta t$ is derived. The analysis is provided along with numerical examples and simulations for a symmetrical layout and uniform speed distribution. Results shed light on how useful more advanced strategies can be.
\end{abstract}

Keywords: Handoff probability forecast, Random Waypoint mobility model, Analytical framework

\section{Introduction}

Users in a cellular network move while their connections are transferred between access points. This process is known as handoff $(\mathrm{HO})$. This same concept holds for different technologies (e.g., GSM, UMTS, LTE, WiFi, ZigBee, WiMAX), although the name of the key element changes according to the specific standard (e.g., base station-BS, node $\mathrm{B}$, access point-AP). As the user moves, the quality of the signal received from the current cell may decrease under some acceptable threshold. This event will trigger the $\mathrm{HO}$ procedure [1]. The time needed to transfer the ongoing communications to the new cell is crucial $[2,3]$ since if this process lasts too long the user may suffer degradation in the quality of the ongoing services. In some cases, it may lead to a drop in the communication [4]. This issue is particularly important for delay-sensitive applications such as video and audio streaming.

Mobility prediction techniques allow the network to prepare for the HO in advance (e.g., booking resources for the expected $\mathrm{HO}$ in the destination cell) [5]. Resource demands could fluctuate abruptly due to the

\footnotetext{
* Correspondence: enrica@entel.upc.edu

Telematics Engineering Department, Universitat Politècnica de Catalunya (UPC), Barcelona, Spain
}

movement of high data rate users. If one can predict requests of bandwidth at a cell, the overall network performance would improve. The goal of our research is to provide a mathematical framework through which the percentage of $\mathrm{HO}$ for users moving with a known mobility pattern can be forecasted. By properly handling this information at the BSs, the network can better manage the resources and prepare the HOs, hence reducing the time needed to authenticate in the connection in the new cell and decreasing the probability of dropping the connection. However, how this prediction is used by the network is out of the scope of this article.

Structural approaches study mobility through mathematical models that try to represent human or vehicle mobility. Mobility models play a key role when planning a wireless network (i.e., resource allocation, location updating, and channel holding time). The Random Waypoint (RWP) mobility model [6] is simple and has extensively been used in simulation studies. Kurkowski et al. [7] analyze MANET simulation studies published in a premiere conference for the MANET community between 2000 and 2005 . They found that $66 \%$ of the studies involving mobility used the RWP mobility model. Despite it has been criticized for not being representative of how humans 
actually move, it is still largely used in many studies [813]. Rojas et al. [14] validate the RWP against real mobility data. With small changes to the distributions used in the RWP (e.g., non-uniform distribution of the waypoints), the authors show that it can be used as a good model for mobility in large geographic areas such as a city. Considering that extracting a mobility pattern from real traces is complex and, anyway, it would be specific to the environment and conditions from which it has been extracted, the use of the RWP in simulation studies is widely accepted. Many researchers have studied the impact of the RWP on routing protocols $[15,16]$, connectivity probabilities $[17,18]$, cell residence time [19-21], and cell change rate $[20,22]$. However, prediction of the probability of $\mathrm{HO}$ to a given cell for users moving according to the RWP mobility pattern has not yet been addressed.

In this article, a detailed description of the analytical framework to forecast the next cell to which a user HOs within a certain period of time is provided. The framework was partially presented in [23]. This analysis follows previous research on statistical characteristics of the RWP mobility model (i.e., length and duration of the straight movement between two waypoints [22], node distribution $[24,25])$. Moreover, simulation results are shown in this study in order to validate the analytical model and check the small impact of the necessary simplifications done. The importance of this study is twofold. First, it provides a deeper insight into the statistics of the RWP mobility model, extending previous analysis [22,24-26]. This statistical knowledge provides a better understanding of the interplay of the mobility pattern with network parameters. Second, and from a more practical perspective, the $\mathrm{HO}$ prediction is useful to manage resource allocation and reservation strategies. According to our model, few requirements are needed to perform the forecast of a possible $\mathrm{HO}$ in the near future: knowledge of the cells' locations and geometries; knowledge of user's position (e.g., through GPS); knowledge of when and where the user changed direction of movement and speed for the last time (e.g., through inertial sensors). Despite the model has been applied to a 4-cell scenario (see Section 4.3), this methodology can be generalized to any symmetrical layout in which fixed BSs are located at the vertices of any regular polygon. Researchers can take advantage of the prediction framework presented in this article for their simulations. As an example, it can be applied in studies on allocation strategies for QoS improvement in cellular networks.

The remainder of this article is organized as follows. Section 2 provides a brief overview of the related literature. The RWP mobility model is explained in Section 3. The method proposed to estimate the probability of $\mathrm{HO}$ to a given cell is shown in Section 4. The method is then applied to different cases: numerical results are provided in Section 5, while simulation results in Section 6 . Section 7 summarizes the main conclusion.

\section{Related work}

A lot of research has been conducted to propose schemes that try to decrease the $\mathrm{HO}$ latencies. This problem is particularly important in IEEE 802.11 networks where it has been proven that these latencies are especially high [2]. Since the scanning time needed to find a new candidate AP is the most constraining phase, many proposals can be find which aim to reduce it. Velayos and Karlsson [3] suggest reducing the link-layer detection time to three consecutive lost frames and to use different timers for the active scanning, thus reducing the search time at least by $20 \%$. An HO procedure is presented in [27], which reduces the discovery phase using a selective scanning algorithm and a caching mechanism. Modification in the standard active scan is proposed in [28]; a user should be able to periodically probe in the background for APs on other channels even when it is already associated with an AP. These make-before-brake algorithms lead to a significant reduction in MAC layer $\mathrm{HO}$ overhead. Similarly, in order to limit the frequency of channel scanning, an adaptive mechanism is recommended in [29] to dynamically adjust the threshold triggering the channel scanning operations. In addition to horizontal HO, i.e., $\mathrm{HO}$ between cells within the domain of a single wireless access technology, recent interest has been directed toward vertical $\mathrm{HO}$ (VHO), which allows users to $\mathrm{HO}$ among heterogeneous wireless access networks [30,31]. Lee et al. [32] investigate the problem of VHO from a $3 \mathrm{G}$ cellular network to a WLAN hotspot. They propose a call admission control that allows the WLAN to limit the VHOs and reduce unnecessary VHO processing. Ben Ali and Pierre [33] evaluate the impact of $\mathrm{VHO}$ on the performance of the voice admission control in different mobility environments.

Some research has been targeted to develop models that predict mobile user trajectory, which can be used to allocate resources at the new cell and to aid the $\mathrm{HO}$ process. Pathirana et al. [34] propose the use of an extended Kalman filter in the prediction of a mobile user's arrival to the next cell. The technique proposed in [5] uses real-time mobile positioning information and previous HO locations reported by other nodes in order to predict the $\mathrm{HO}$ event. With mobility prediction, the reservation at each BS can dynamically be adjusted. They demonstrated that reservation efficiency improves as the knowledge incorporated into the scheme increases. Joshi et al. [35] aim to exploit the notion of predictability and propose algorithms to achieve low latencies while minimizing the message overhead. The prediction technique proposed in [13] uses both simple moving average for (sub-)inertial movements and simple mobility pattern matching for non-inertial movements. An $\mathrm{HO}$ prediction 
algorithm is designed for eNodeB's HO preparation, thus improving the $\mathrm{HO}$ in $3 \mathrm{G}$ LTE systems.

Prediction of users' movement is also possible using trace data [36]. For $\mathrm{HO}$ success, the most important predictions occur when moving into highly populated cells. Other studies exploit real traces to analyze the predictability of users' movements inside the network [37,38]. Sricharan and Vaidehi [37] examine real-time mobility traces and identify key mobility parameters. A generic framework for mobility prediction is proposed in [38] where a model is proposed to predict the sequences of a user's path from observed sequences. Libo et al. [39] conducted an extensive empirical comparison of four important classes of location predictors in order to prove which could most accurately predict the next cell for a mobile wireless-network user. They found that the complex predictors were at best only negligibly better than the simple Markov predictor.

Much effort has been made to gain understanding of the implications that the use of the RWP model may cause. The "steady-state" problem has been addressed in $[22,40]$. By giving a formal description of the RWP model in terms of a discrete-time stochastic process, Bettstetter et al. [22] investigate the length and time of the movement between waypoints, the spatial distribution of nodes, the direction angle at a new waypoint, and the cell change rate. Navidi and Camp [40] derive the stationary distributions for location, speed, and pause time. The spatial node distribution of nodes is also addressed in $[24,25]$. Given that the RWP tends to bring the nodes to the centre of the area, Bettstetter et al. [24] proposed to initially distribute the nodes according to a given pattern so that the network starts in the steady state. Hyttia et al. [25] propose a general expression for the node distribution without using approximations. An exact formula for the mean arrival rate across an arbitrary curve is presented in [20]. This result, together with the distribution of a node's location, allows the computation of other metrics, such as the mean $\mathrm{HO}$ rate and the mean dwell time. Pla and Casares-Giner [19] propose a model of the sojourn time in the overlap area for users moving according to the RWP. To simplify their study, they assume that waypoints cannot fall inside the overlap area. The obtained results show that the common assumption of exponential distribution for this time is not suitable.

\section{Random waypoint mobility model}

Modeling the mobility of terminals in wireless networks, where the connection has to be handled through several APs, is a key in order to forecast the quality of service and design the necessary resources. In general, high mobility leads to poorer quality of service since more $\mathrm{HO}$ that cause extra overhead are performed. While it is obvious that faster speeds tend to cause more $\mathrm{HO}$, it must be highlighted that the mobility pattern is also related to the $\mathrm{HO}$ rate: a terminal moving in circles close to the $\mathrm{AP}$ at high speed may not require $\mathrm{HO}$ while another one moving away from the AP will certainly require an $\mathrm{HO}$ soon.

The RWP was introduced in [6]. In this model, each node is assigned an initial location $\left(\mathrm{WP}_{0}\right)$ and a destination $\left(\mathrm{WP}_{1}\right)$, which are chosen independently and uniformly within the whole area (i.e., the area where nodes can move, which is generally assumed to be convex). A speed $(v)$ is assigned as well, which is chosen uniformly or according to any other distribution from an interval $\left(v_{\min }\right.$ to $\left.v_{\max }\right)$ and independent of the initial location and destination. Between the two waypoints $\mathrm{WP}_{0}$ and $\mathrm{WP}_{1}$, the node follows a straight line and moves at a constant speed. The movement between two waypoints is referred to as a leg or transition. After reaching $\mathrm{WP}_{1}$, a new destination and a new speed are chosen independent of previous destinations and speeds. The node may remain paused for some random pause time before starting its movement toward the next waypoint. For simplicity, pause times are not considered in this study.

Exact formulas for the probability density function (pdf) of the distance between two waypoints, i.e., pdf of the transition length, $f_{L}(\ell)$ in different scenarios are provided in $[22,25]$. According to the geometry and dimensions of the area, the mean distance can be evaluated.

\section{Probability of handoff to a given cell}

\subsection{Problem statement}

The objective of the study is to estimate the probability of $\mathrm{HO}$ to a given cell before a given period of time $(\Delta t)$ elapses, provided that we know where and when the last waypoint was reached. The forecast is performed under the following assumptions:

- The whole area $A$ is a circle of radius $R$, and APs are placed at the corners of a regular polygon inside the circle. Any regular polygon can be considered (e.g., a square as shown in Section 4.3).

- Ideal conditions are considered (i.e., noise and fading are not taken into account).

- Coverage areas have a circular shape.

- Current position $P_{t}$ at time $t$ is known.

- Position and time of the last waypoint $\left(\mathrm{WP}_{j}\right.$ at time $\left.j\right)$ are known (i.e., where and when the node changed to the present direction and speed). Alternatively, movement direction and time of the last waypoint can be used to derive the position of the last waypoint.

- It is assumed that the number of waypoints $X(\Delta t)$ that a node may reach during $\Delta t$ is smaller or equal to one.

- In general, the node may perform multiple HOs in $\Delta t$; in this study, only the first $\mathrm{HO}$ (if any) is studied. 


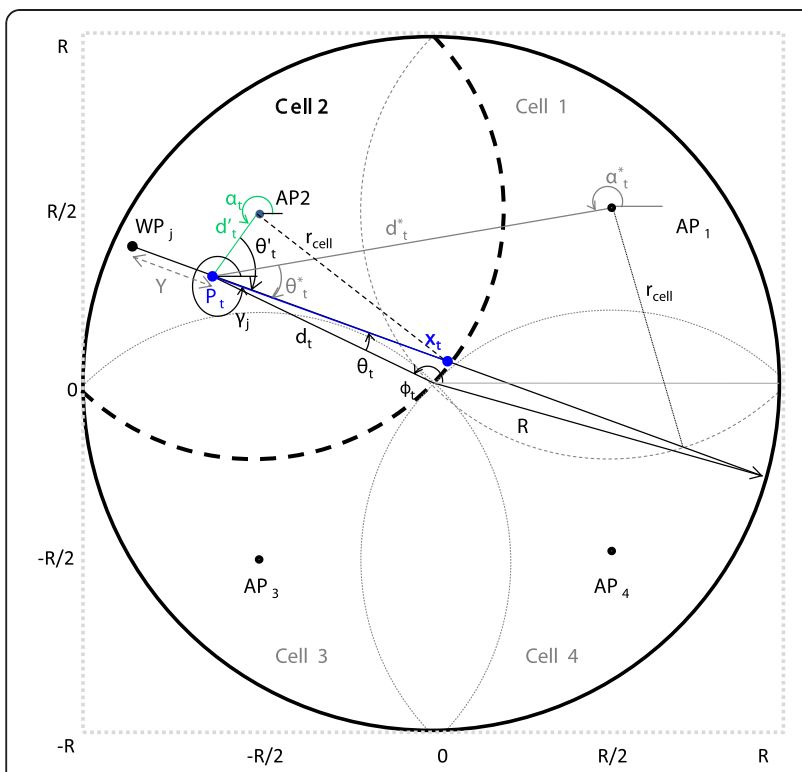

Figure 1 Layout with four APs. Current cell is cell 2 (AP2). The distance to the cell boundary $\left(x_{t}\right)$ in current direction $\gamma_{j}$ is displayed, together with other parameters used in the analysis.

- When the node is inside the coverage areas of two or more APs (i.e., overlapping area), the HO starts when the node exits the coverage area of the AP to which it is currently associated. Other HO strategies may be applied, with minor changes in the proposed framework.
The following parameters are considered (a layout with four APs is depicted in Figure 1 for better understanding):

- $\gamma_{j}$ represents the current angle of direction and $v_{j}$ the current speed.

- $Y$ is the distance between $\mathrm{WP}_{\mathrm{j}}$ and $P_{t}$ (i.e., distance already traveled at speed $v_{j}$ in direction $\gamma_{j}$ ).

- $x_{t}, x_{A}$, and $x_{t A P}$ represent the distances from $P_{t}$ to the cell boundaries, the boundaries of $A$ and the borders of the neighboring cell in direction $\gamma_{j}$, respectively (see Figures 1 and 2). The neighboring cell is the cell toward which the MN is moving in direction $\gamma_{j}$.

- If there are two intersections with the neighboring cell, they are identified with $x_{t A P+}$ and $x_{t A P-}$ (see Figure $2 \mathrm{~b}$ ).

The definition of a node's movement direction $\gamma_{j}$ in a two-dimensional space can be better understood through Figure 1. As shown in [22], the outcome of this angle depends on both polar coordinates of $P_{t}$ (e.g., $d_{t}$ and $\left.\Phi_{t}\right)$. Thus, a second alternative direction angle $\theta_{t}$ is introduced. This angle is defined in a way that $\theta_{t}=0$ for movements going through the center of the circular area. A major advantage of this definition is that the outcome of $\theta_{t}$ is independent of the polar angle $\Phi_{t}$. It can directly be mapped onto $\gamma_{j}$ for a given $\Phi_{t}$. If we define that counterclockwise angles count positive

$$
\gamma_{j}=\theta_{t}+\phi_{t}+\pi
$$

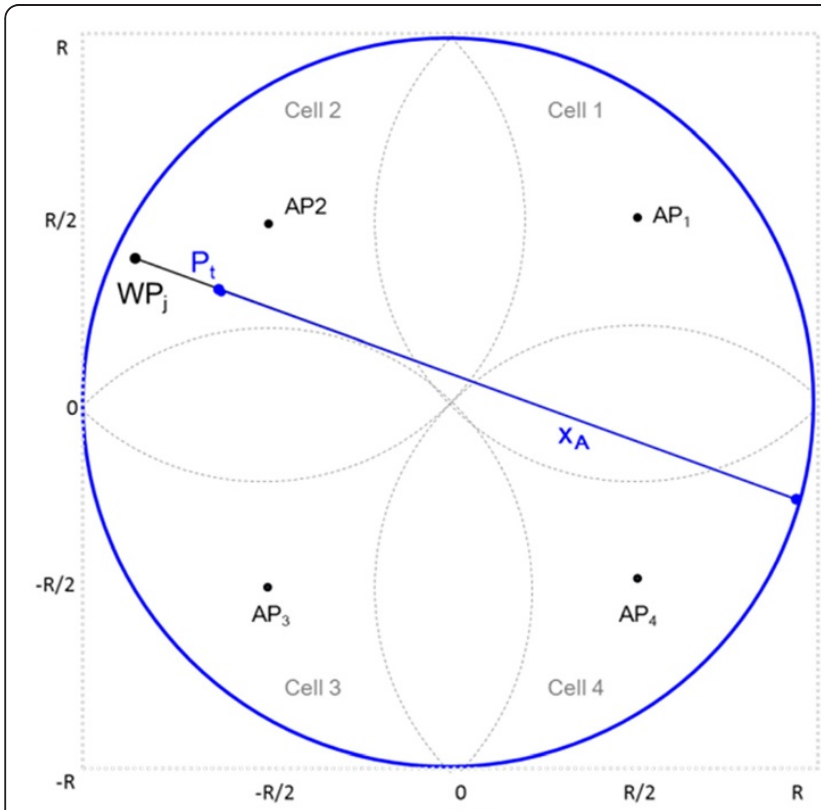

(a)

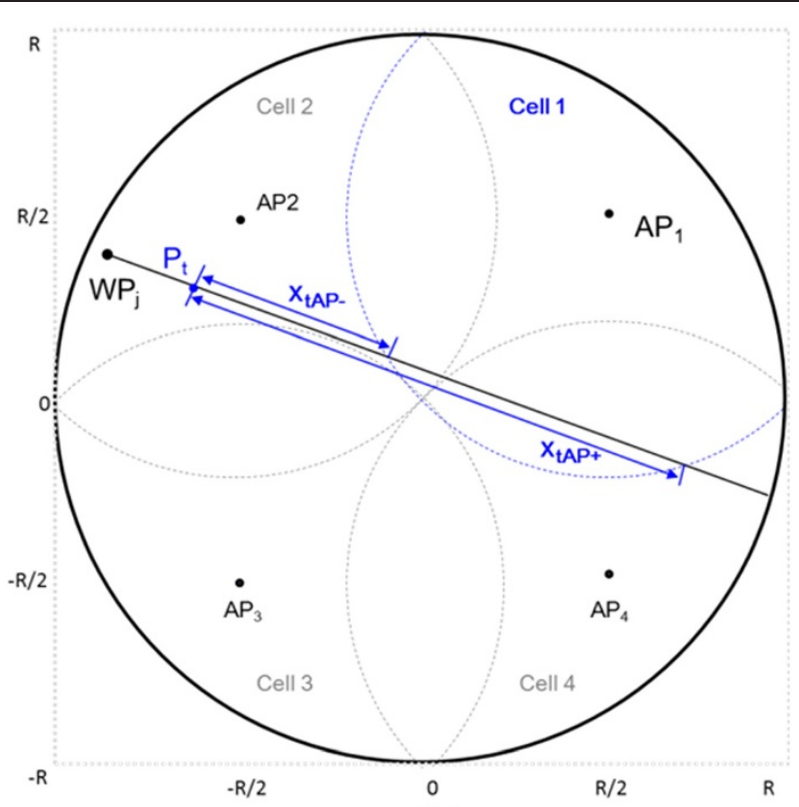

(b)

Figure 2 Layout with four APs. The distances from the whole area $\left(x_{A}\right)$ and neighbouring cell $\left(x_{t A P}\right)$ in current direction $\gamma_{j}$ are displayed in (a) and (b), respectively. Neighboring cell is cell 1 (AP1). 
In a circular layout, the formula for the maximum length of the movement from $P_{t}$ in direction $\gamma_{j}$ (i.e., $x_{A}$ according to previous definition) is [22]

$$
x_{A}\left(\theta_{t}\right)=d_{t} \cdot \cos \theta_{t}+\sqrt{R^{2}-\left(d_{t} \cdot \sin \theta_{t}\right)^{2}}
$$

where $d_{t}$ and $\Phi_{t}$ are the polar coordinates of current position $P_{t}$ with respect to the center of $A$. Similarly

$$
\begin{aligned}
& x_{t}\left(\theta_{t}^{\prime}\right)=d_{t}^{\prime} \cdot \cos \theta_{t}^{\prime}+\sqrt{r_{\text {cell }}^{2}-\left(d_{t}^{\prime} \cdot \sin \theta_{t}^{\prime}\right)^{2}}, \\
& \left\{\begin{array}{l}
x_{t A P+}\left(\theta_{t}^{*}\right)=d_{t}^{*} \cdot \cos \theta_{t}^{*}+\sqrt{r_{\text {cell }}^{2}-\left(d_{t}^{*} \cdot \sin \theta_{t}^{*}\right)^{2}} \\
x_{t A P-}\left(\theta_{t}^{*}\right)=d_{t}^{*} \cdot \cos \theta_{t}^{*}-\sqrt{r_{\text {cell }}^{2}-\left(d_{t}^{*} \cdot \sin \theta_{t}^{*}\right)^{2}}
\end{array}\right.
\end{aligned}
$$

where $d_{t}^{\prime}$ is the distance of $P_{t}$ to the center of the current cell, $\theta_{t}^{\prime}=\gamma_{j}-\alpha_{t}-\pi$ (see Figure 1), $\alpha_{t}$ is the angle of $P_{t}$ with respect to the center of the cell, $r_{\text {cell }}$ is the radius of the cell, $d_{t}^{*}$ is the distance of $P_{t}$ to the center of the neighboring cell (i.e., cell 1 in Figure 2), $\theta_{t}^{*}=\gamma_{j}-\alpha_{t}^{*}-\pi$, and $\alpha_{t}^{*}$ is the angle of $P_{t}$ with respect to the center of the neighboring cell. From (1), we can write

$$
\begin{aligned}
& \theta_{t}^{\prime}=\theta_{t}+\phi_{t}-\alpha_{t}, \\
& \theta_{t}^{*}=\theta_{t}+\phi_{t}-\alpha_{t}^{*} .
\end{aligned}
$$

\subsection{Probability of handoff}

Given the number of waypoints $X(\Delta t)$ that a node may reach during $\Delta t$, the probability of $\mathrm{HO}$ can be written as

$$
\begin{aligned}
\operatorname{Pr}\{\mathrm{HO} \text { in } \Delta t\}= & \sum_{i=0}^{\infty} \operatorname{Pr}\{\mathrm{HO} \text { in } \Delta t \mid X(\Delta t)=i\} . \\
& \operatorname{Pr}\{X(\Delta t)=i\} .
\end{aligned}
$$

According to initial assumptions, $\operatorname{Pr}\{X(\Delta t)=i\}$ is zero for $i>1$. Thus, Equation (7) can be written as

$$
\begin{aligned}
\operatorname{Pr}\{\mathrm{HO} \text { in } \Delta t\}= & \operatorname{Pr}\{\mathrm{HO} \text { in } \Delta t \mid X(\Delta t)=1\} \cdot \operatorname{Pr}\{X(\Delta t)=1\} \\
& +\operatorname{Pr}\{\mathrm{HO} \text { in } \Delta t \mid X(\Delta t)=0\} \cdot \operatorname{Pr}\{X(\Delta t)=0\} \\
= & \operatorname{Pr}\{\mathrm{HO} a\} \cdot \operatorname{Pr}\{X(\Delta t)=1\} \\
& +\operatorname{Pr}\{\mathrm{HO} b\} \cdot(1-\operatorname{Pr}\{X(\Delta t)=1\}),
\end{aligned}
$$

where $\operatorname{Pr}\{X(\Delta t)=1\}$ represents the probability of reaching the waypoint during $\Delta t, \operatorname{Pr}\{\mathrm{HO} a\}$ is the probability of $\mathrm{HO}$ given that a waypoint is reached during $\Delta t$, while $\operatorname{Pr}\{\mathrm{HO} b\}$ is the probability of $\mathrm{HO}$ given that node moves on same direction and at same speed during $\Delta t$. Because the current direction of movement $\gamma_{j}$ and the current speed $v_{j}$ are known, $\operatorname{Pr}\{\mathrm{HO} b\}$ only depends on the distance to the cell border in direction $\gamma_{j}$ and can easily be calculated.

The flow charts in Figures 3 and 4 show the algorithm used to estimate the probability of $\mathrm{HO}$, the probability of no $\mathrm{HO}$, and the expressions used in each case. Depending on the node's direction of movement, the distance of $P_{t}$ to $A\left(x_{A}\right)$ is longer or shorter than the distance of $P_{t}$ to the cell boundaries $\left(x_{t}\right)$ (i.e., the node is moving towards the

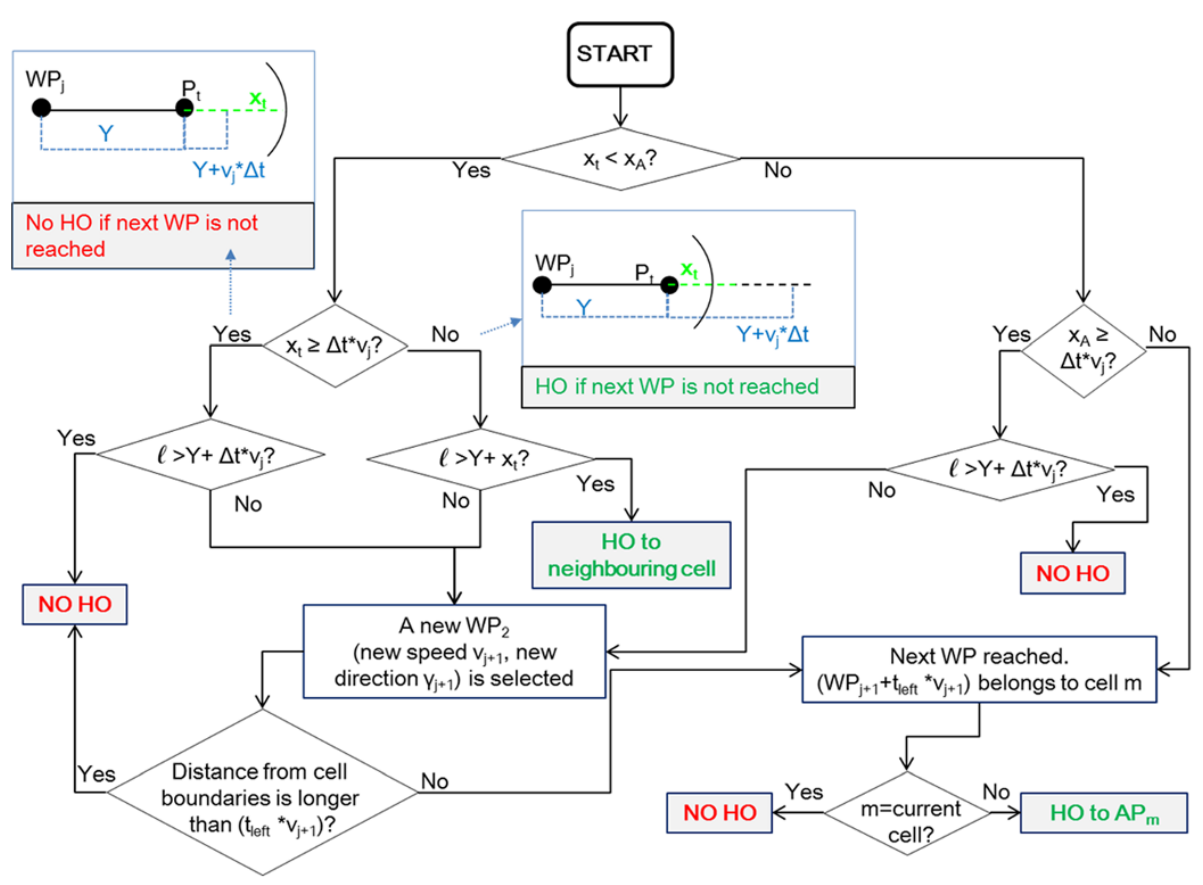

Figure 3 Flow chart for estimation of the probability of $\mathrm{HO}$ or no $\mathrm{HO}$. 


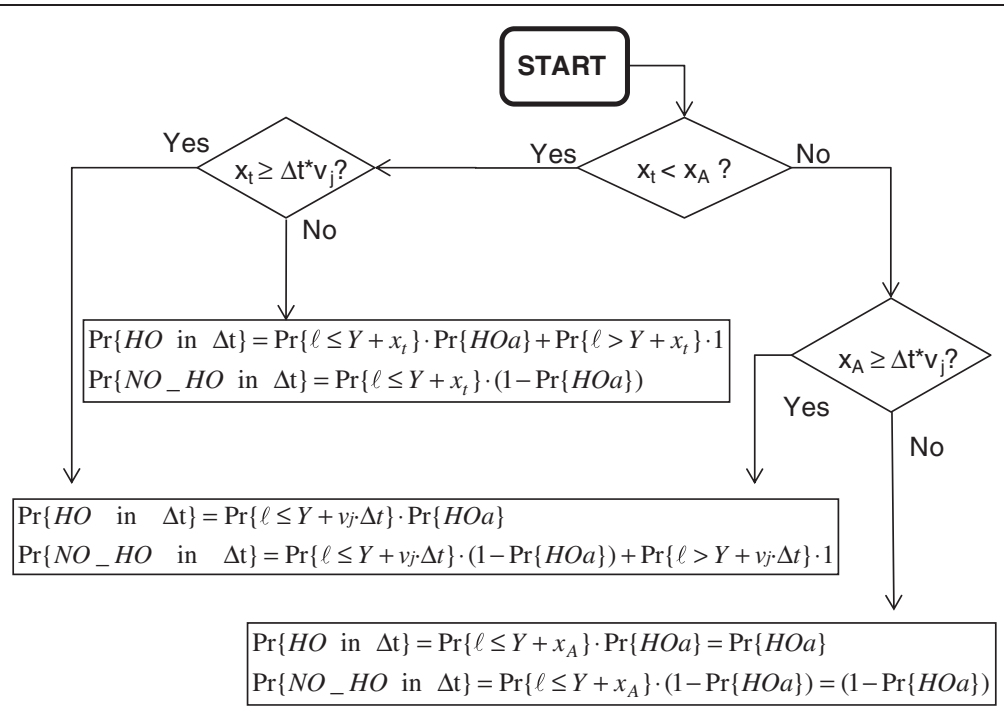

Figure 4 Formulas used to determine the probability of $\mathrm{HO}$ and no $\mathrm{HO}$.

center of $A$ or in opposite direction, respectively). Figure 3 shows that, in the first case $\left(x_{t}<x_{A}\right)$, an $\mathrm{HO}$ is performed if the distance to the cell boundaries is shorter than the distance walked at given speed during $\Delta t\left(x_{t}<v_{j} \ldots \Delta t\right)$ and the distance between two waypoints $(\ell)$ is longer than $Y+x_{t}$ (i.e., the node does not reach the waypoint before exiting its current cell, thus $\mathrm{Pr}$ $\{\mathrm{HO} b\}$ is one). On the other hand, if $x_{t} \geq x_{A}$, then an $\mathrm{HO}$ is performed only if the distance to the boundaries of $A$ is shorter than the distance walked at given speed during $\Delta t\left(x_{A}<v_{j} \ldots \Delta t\right)$ and the point reached in the new direction falls outside the current cell. In all the other cases, the node will remain inside its current cell. In Figure 4, the corresponding expressions for each case are provided.

In the following sections, expressions for the probability of reaching the next waypoint and for $\operatorname{Pr}\{\mathrm{HO} a\}$ are provided.

\subsubsection{Probability of reaching the next waypoint during $\Delta t$}

Recall that the node is located at $P_{t}$ at time $t$ and that it has been traveling a distance $Y$ from previous waypoint $\mathrm{WP}_{j}$. The next waypoint may be located at any point on the straight line between $P_{t}$ and $P_{t}+v_{j}$ - $\Delta t$ (if $x_{t} \geq v_{j} \cdot \Delta t$ and $x_{t}<x_{A}$ ), between $P_{t}$ and $P_{t}+x_{t}$ (if $x_{t}<v_{j} \cdot \Delta t$ and $x_{t}<x_{A}$ ), or between $P_{t}$ and $P_{t}+x_{A}$ (if $x_{t} \geq x_{A}$ ). We define this generic point on the current direction of movement at generic distance $r$ from $P_{t}$ as $S(r)$ (i.e., $S(r=0) \equiv P_{t}$ ). The expression $\operatorname{Pr}\{X$ $\left.\left(t+r / v_{j}\right)=1\right\}$ represents the probability of reaching the next waypoint after traveling a distance $r$ at current speed $v_{j}$, where $t+\frac{r}{v_{j}} \leq \Delta t$. This probability can be rewritten as the probability that the distance between two waypoints $(\ell)$ is shorter than a generic distance $L=Y+r$. Thus, depending on the geometry and on the current speed, we find different expressions

$$
\begin{aligned}
& \operatorname{Pr}\left\{l \leq Y+x_{t}\right\}=\int_{Y}^{Y+x_{t}\left(\theta_{t}^{\prime}\right)} f_{L}\left(l \mid \theta_{t}\right) d l \text { if } x_{t}\left(\theta_{t}^{\prime}\right)<v_{j} \cdot \Delta t \text { and } x_{t}\left(\theta_{t}^{\prime}\right)<x_{A}\left(\theta_{t}\right) \\
& \operatorname{Pr}\left\{l \leq Y+x_{A}\right\}=\int_{Y}^{Y+x_{A}\left(\theta_{t}\right)} f_{L}\left(l \mid \theta_{t}\right) d l \text { if } x_{A}\left(\theta_{t}\right)<v_{j} \cdot \Delta t \text { and } x_{t}\left(\theta_{t}^{\prime}\right) \geq x_{A}\left(\theta_{t}\right) \\
& \operatorname{Pr}\left\{l \leq Y+v_{j} \cdot \Delta t\right\}=\int_{Y}^{Y+v_{j} \cdot \Delta t} f_{L}\left(l \mid \theta_{t}\right) d l \text { if } x_{t}\left(\theta_{t}^{\prime}\right) \geq v_{j} \cdot \Delta t \text { or } x_{A}\left(\theta_{t}\right) \geq v_{j} \cdot \Delta t
\end{aligned}
$$


where $f_{L}\left(\ell \mid \theta_{t}\right)$ is the pdf of the distance between two waypoints provided that the current direction of movement is known. According to [22], if $A$ is a circle of radius $R, f_{L}\left(l \mid \theta_{t}\right)=\frac{8}{\pi * R} \cdot \frac{l}{2 \cdot R}\left(\arccos \left(\frac{l}{2 \cdot R}\right)-\frac{l}{2 \cdot R} \cdot \sqrt{1-\left(\frac{l}{2 \cdot R}\right)^{2}}\right)$.

\subsubsection{Probability of $\mathrm{HO}$ when the waypoint is reached (Pr\{HOa\})}

Consider that the node reaches the next waypoint at the generic point $S(r)$ at time $\left(t+\frac{r}{v_{j}}\right)$. We can define $t r$ as the remaining time before $\Delta t$ elapses. It can take values between $(0, \Delta t)$. We can write

$$
t_{r}=\Delta t-\frac{r}{v_{j}}
$$

The probability that the node reaches another waypoint during $t_{r}$ is small when $\Delta t$ is small. According to our initial assumption, by accurately choosing $\Delta t$, we can discard the possibility that another waypoint is reached, which would highly complicate the solution. The polar coordinates of the waypoint are $\left(d_{\mathrm{WP}}, \Phi_{\mathrm{WP}}\right)$. At this point, a new waypoint will randomly be selected inside $A$ and the node will start moving in a new direction. According to Bettstetter et al. [22], nodes take a non-uniformly distributed angle of direction at each new waypoint. The pdf of this angle depends on the shape of $A$ and the starting waypoint of the node. Thus, in a circular area of radius $R$, the pdf of the angle of direction $\gamma$ is

$$
f_{\Gamma}\left(\gamma \mid\left(d_{\mathrm{WP}}, \phi_{\mathrm{WP}}\right)\right)=f_{\Theta}\left(\theta \mid d_{\mathrm{WP}}\right)=\frac{1}{2 \pi R^{2}} x_{A}^{2}\left(\theta_{t}\right) .
$$

Moreover, a new speed will randomly be selected at the waypoint. The points reached after $t_{r}$ at maximum and minimum speed are estimated in each possible direction of movement. These points identify a crown where the node may be located after $\Delta t$. Since the speed is uniformly distributed between $v_{\min }$ and $v_{\max }$, each point among $\left(v_{\min } \cdot t_{r}\right)$ and $\left(v_{\max } \cdot t_{r}\right)$ is equally probable. Each direction may be selected according to (11). The area where the node may move can be divided into two areas: one inside the current cell (i.e., no $\mathrm{HO}$ is performed) and another one inside one (at least) of the neighboring cells (i.e., an $\mathrm{HO}$ is performed). According to previous definitions, the latter is $\operatorname{Pr}\{\mathrm{HO} a \mid r\}$. As $S(r)$ moves away from $P_{t}$, the radius of the crown will decrease and the distances and angles will change. Thus, we have to evaluate $\operatorname{Pr}\{\mathrm{HO} a \mid r\}$ at any distance $r$ from $P_{t}$. The final expression to estimate the probability of $\mathrm{HO}$ given that the waypoint is reached during $\Delta t$ is

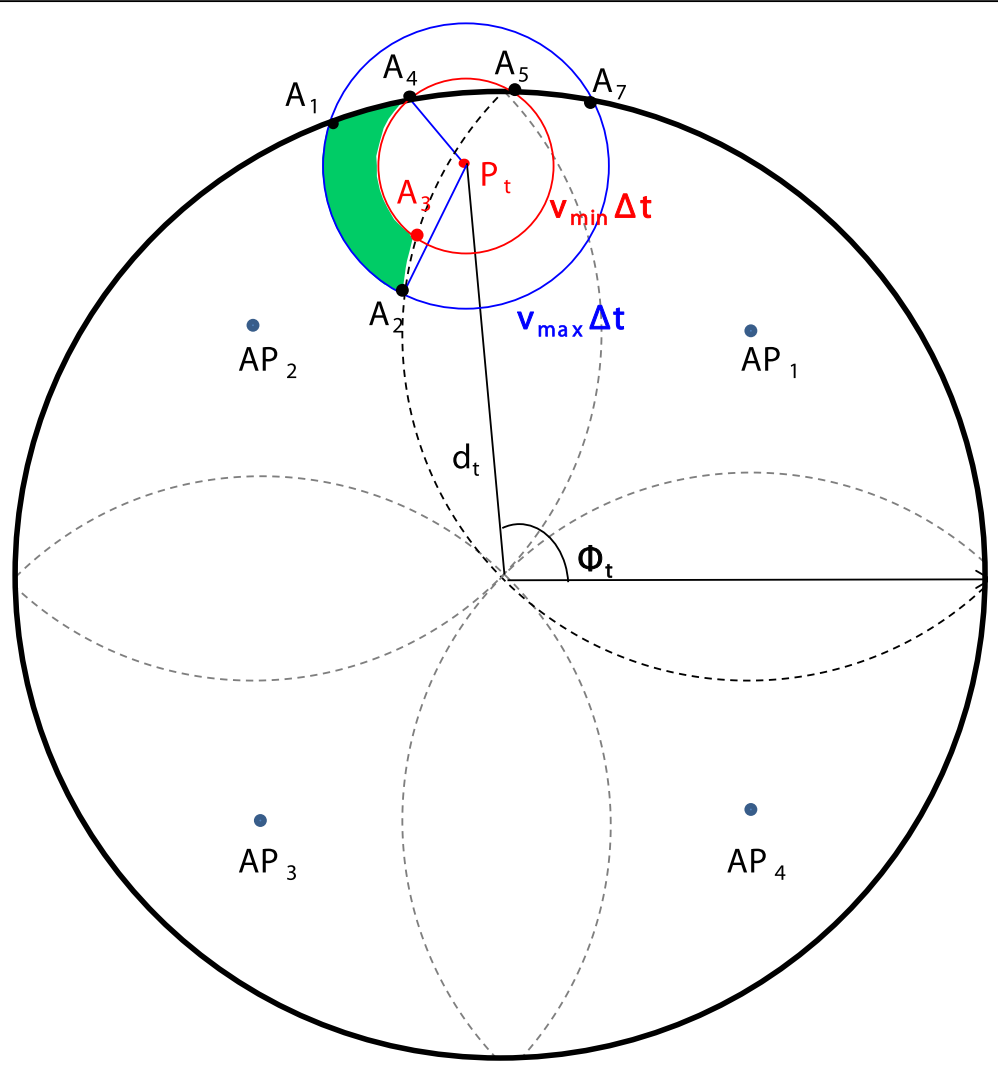

Figure 5 Area representing the probability of $\mathrm{HO}$ if the next waypoint is $\boldsymbol{P}_{t}$ (i.e., $\operatorname{Pr}\{\mathrm{HO} a \mid r=0\}$ ). The node is currently associated with AP1. 


$$
\operatorname{Pr}\{\mathrm{HO} a\}=\left\{\begin{array}{l}
\int_{0}^{v_{j} \cdot \Delta t} \operatorname{Pr}\{H O a \mid r\} \cdot f_{R}(r) d r \text { if } x_{t}\left(\theta_{t}^{\prime}\right) \geq v_{j} \cdot \Delta t \text { and } x_{t}\left(\theta_{t}^{\prime}\right)<x_{A}\left(\theta_{t}\right) \\
\int_{0}^{x_{t}\left(\theta_{t}^{\prime}\right)} \operatorname{Pr}\{H O a \mid r\} \cdot f_{R}(r) d r \text { if } x_{t}\left(\theta_{t}^{\prime}\right)<v_{j} \cdot \Delta t \text { and } x_{t}\left(\theta_{t}^{\prime}\right)<x_{A}\left(\theta_{t}\right) \\
\int_{0}^{x_{A}\left(\theta_{t}\right)} \operatorname{Pr}\{H O a \mid r\} \cdot f_{R}(r) d r \text { if } x_{t}\left(\theta_{t}^{\prime}\right) \geq x_{A}\left(\theta_{t}\right),
\end{array}\right.
$$

where $\operatorname{Pr}\{\mathrm{HO} a \mid r\}$ represents the probability of $\mathrm{HO}$ given that the next waypoint is $S(r)$, and $f_{R}(r)$ is the pdf of the possible positions $S(r)$. Note that the waypoints are uniformly selected in $A$.

\subsection{Application to four APs}

In order to illustrate how the analytical framework provides the forecast of the $\mathrm{HO}$ in a specific scenario, we will consider a layout with four APs. The coverage area of each AP is a circular area of radius rcell. The APs are located at the vertices of a square whose side is $R$, as shown in Figure 1. In Figure 5, the method to calculate the probability of $\mathrm{HO}$ when the next waypoint is $P_{t}$ (i.e., $\operatorname{Pr}\{\mathrm{HO} a \mid r=0\}$ ) is illustrated. From the current point $P_{t}$, a circular crown can be drawn with inner radius $\left(v_{\min } \cdot \Delta t\right)$ and outer radius $\left(v_{\max } \cdot \Delta t\right)$. The crown represents the area where the node may be located if it changes its direction of movement and speed at $P_{t}$. Because the speed distribution is uniform between $v_{\min }$ and $v_{\max }$, the distribution of the node locations' inside this area is uniform too. Points inside the crown but outside $A$ are discarded (i.e., they fall outside the area of movement). Angles for the points $A 1, A 4, A 5$, and $A 7$ should be estimated; we refer to these angles as $\theta_{A 1}, \theta_{A 4}$,

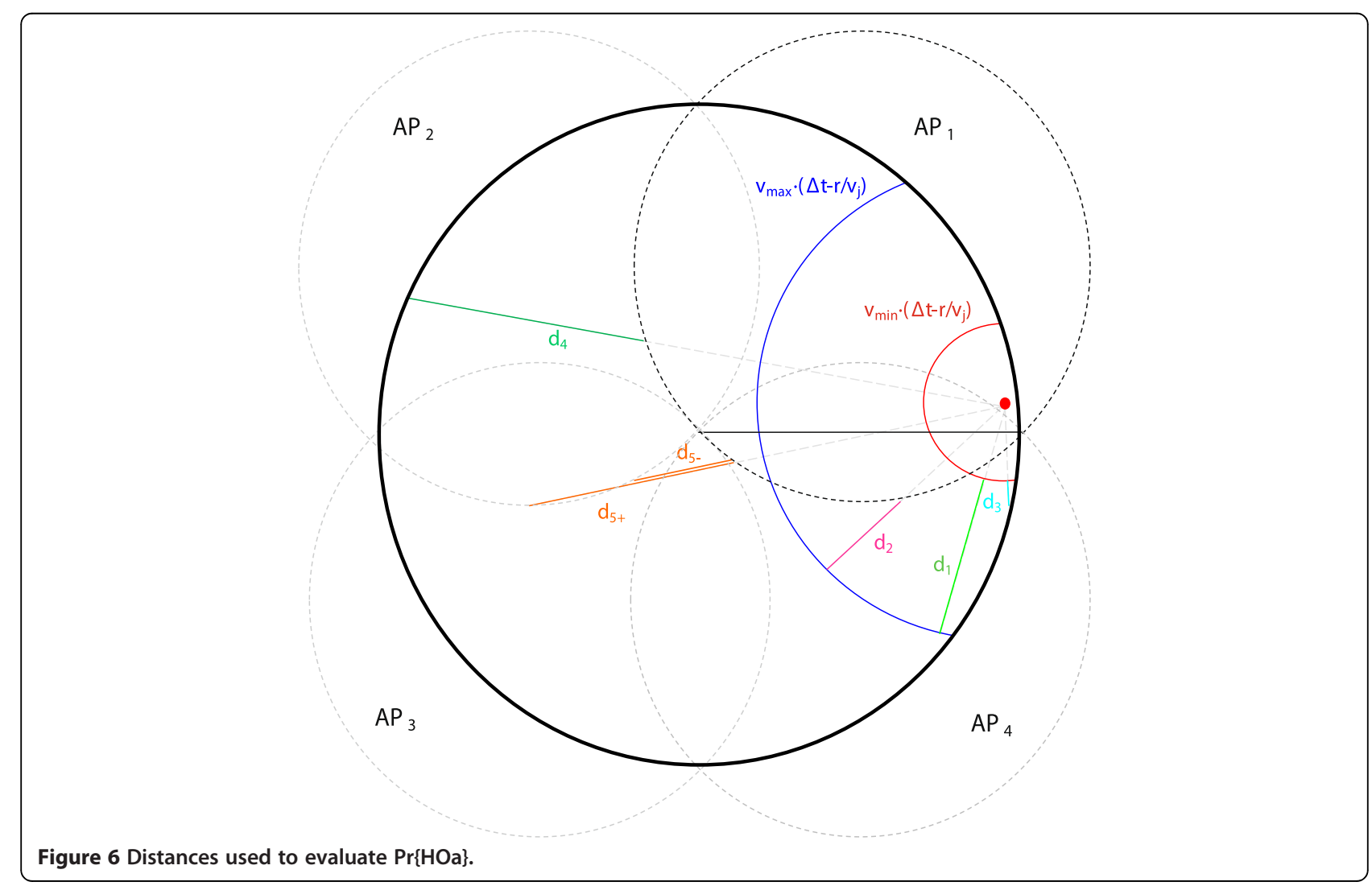




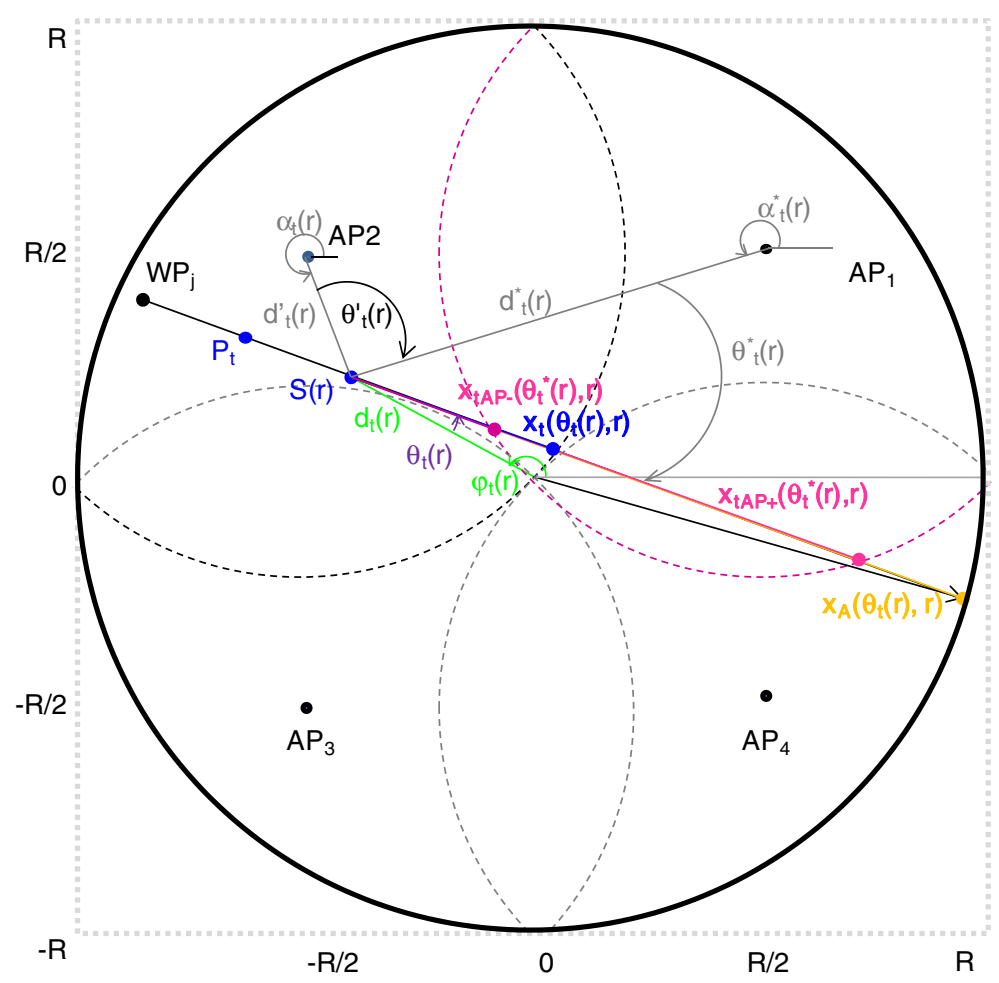

Figure 7 Distances from the cell boundary $\left(x_{t}\right)$, whole area $\left(x_{A}\right)$, and neighboring cell $\left(x_{t A P}\right)$ in current direction $\gamma_{j}$ at point $S(r)$.

$\theta_{A 5}$, and $\theta_{A 7}$, respectively. The $\mathrm{HO}$ area is represented in green in Figure 5 (i.e., the node is currently associated with AP1). It spans from angle $\theta_{A 4}$ and $\theta_{A 2}$. The probability of $\mathrm{HO}$ in this case equals the probability of $\mathrm{HO}$ to AP2 and can be evaluated as

$$
\begin{aligned}
\operatorname{Pr}\{\mathrm{HO} a \mid r & =0\} \\
& =\int_{\theta A 4}^{\theta A 2} f_{\Theta}\left(\theta \mid d_{t}(r)\right) \cdot \frac{\min (d 1(r), d 2(\theta, r), d 3(\theta, r))}{\min (d 1(r), d 3(\theta, r))}
\end{aligned}
$$

where $r$ is the distance already walked from $P_{t}$ (i.e., zero in this case), $d 1(r)$ is the difference of the two radii of the crown (see Figure 6), $d 2(\theta, r)$ is the difference between the outer radius of the crown and $x_{t}\left(\theta_{t}^{\prime} r\right), d 3(\theta, r)$ is the difference of $x_{A}\left(\theta_{t} r\right)$ and the inner radius of the crown, and $\theta$ represents all possible angles of movement from the current position with respect to the centre of A. $f_{\Theta}\left(\theta \mid d_{t}(r)\right)$ is defined in (11) and represents the pdf of the direction angle $\theta$ provided that we are at distance $d_{t}$ $(r)$ from the centre of $A$.

The integral in (13) cannot be evaluated because the integrand function changes in the interval of integration. Instead, a sum of integrals is used where the minimum function is substituted by the corresponding minimum distance at each interval (see Section 5). Expressions for $d 1(r), d 2(\theta, r)$, and $d 3(\theta, r)$ are provided in Appendix 1 . Depending on the current position of the node, the circular crown intersects the cell boundaries and/or $A$ at different points. All of the other possible distances $(d 4(\theta$, $r), d 5(\theta, r), d 6(\theta, r))$ are provided in Appendix 1. Refer to Figure 6 to better understand these values.

Equation 13) is evaluated iteratively at any point $S(r)$ according to (12). In each iteration, the time decreases as the node moves at speed $v_{j}$ in direction $\gamma_{j}$; then, the radius of the crown will decrease, and the distances and

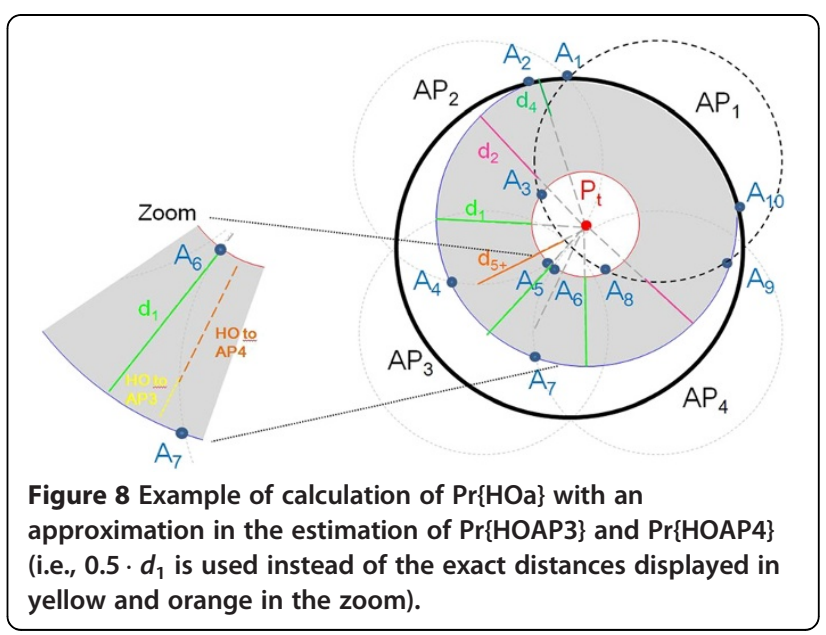


Table 1 Probability $\varepsilon$ with which $X(\Delta t)$ is smaller or equal to one

\begin{tabular}{lcccc}
\hline$\Delta \boldsymbol{t}(\mathbf{s})$ & $\mathbf{5 0}$ & $\mathbf{8 0}$ & $\mathbf{9 0}$ & $\mathbf{1 0 0}$ \\
\hline$\varepsilon(\%)$ & 99.20 & 95.82 & 93.81 & 91.30 \\
\hline
\end{tabular}

angles will change. Equations (2)-(4) for the generic case (i.e., the next waypoint is $S(r)$ ) are provided in Appendix 2. Figure 7 represents the distances previously defined according to $S(r)$.

\section{Numerical examples}

The analysis is implemented with Maple. The integrals in (12) are solved through numerical integration. As mentioned, the integral in (13) is split into a sum of integrals where the minimum function is substituted by the corresponding minimum distance. For that purpose, all possible cases are considered, and all possible combinations of angles are analyzed. As an example, Equation (13) is evaluated as

$$
\begin{aligned}
\operatorname{Pr}\{\mathrm{HO} a \mid r=0\}= & \int_{\theta A 4}^{\theta A 1} f_{\Theta}\left(\theta \mid d_{t}(0)\right) \cdot \frac{d 3(\theta, 0)}{d 3(\theta, 0)} d \theta \\
& +\int_{\theta A 3}^{\theta A 2} f_{\Theta}\left(\theta \mid d_{t}(0)\right) \cdot \frac{d 2(\theta, 0)}{d 1(0)} d \theta \\
& +\int_{\theta A 1}^{\theta A 3} f_{\Theta}\left(\theta \mid d_{t}(0)\right) \cdot \frac{d 1(0)}{d 1(0)} d \theta
\end{aligned}
$$

In a few cases, due to the complexity of the geometry, an approximation is applied in the estimation of the probability of HO. Figure 8 shows an example in which the approximation is used (i.e., for the probability of $\mathrm{HO}$ to AP3 and AP4). These probabilities are computed as

$$
\begin{aligned}
\operatorname{Pr}\{\mathrm{HOAP3a} \mid r=0\}= & \int_{\theta A 4}^{\theta A 5} f_{\Theta}\left(\theta \mid d_{t}(0)\right) \cdot \frac{d 2(\theta, 0)-d 5^{+}(\theta, 0)}{d 1(0)} d \theta \\
& +\int_{\theta A 5}^{\theta A 6} f_{\Theta}\left(\theta \mid d_{t}(0)\right) \cdot \frac{d 1(0)}{d 1(0)} d \theta \\
& +0.5 \cdot \int_{\theta A 6}^{\theta A 7} f_{\Theta}\left(\theta \mid d_{t}(0)\right) \cdot \frac{d 1(0)}{d 1(0)} d \theta \\
\operatorname{Pr}\{\mathrm{HOAP4a|r=}=0\}= & \int_{\theta A 7}^{\theta A 8} f_{\Theta}\left(\theta \mid d_{t}(0)\right) \cdot \frac{d 1(0)}{d 1(0)} d \theta \\
& +\int_{\theta A 8}^{\theta A 9} f_{\Theta}\left(\theta \mid d_{t}(0)\right) \cdot \frac{d 2(\theta, 0)}{d 1(0)} d \theta \\
& +0.5 \cdot \int_{\theta A 6}^{\theta A 7} f_{\Theta}\left(\theta \mid d_{t}(0)\right) \cdot \frac{d 1(0)}{d 1(0)} d \theta,
\end{aligned}
$$

where in both equations the last term stands for the approximation (see zoom in Figure 8).

The time $\Delta t$ is selected such that, with a given probability $\varepsilon$, it is guaranteed that the node will reach no

\begin{tabular}{|c|c|c|c|c|c|}
\hline Cases & 1 & 2 & 3 & 4 & 5 \\
\hline$P_{t}$ & $(1 ; 3 \pi / 4)$ & $(2 ; 7 \pi / 4)$ & $(138 ; 0)$ & $(1 ; 3 \pi / 2)$ & $(138 ; \pi / 4)$ \\
\hline$W P_{j}$ & $(63 ; 7 \pi / 4)$ & $(140 ; 7 \pi / 4)$ & $(138 ; \pi)$ & $(10 ; 3 \pi / 2)$ & $(70 \sqrt{ } 2 ; \pi / 4)$ \\
\hline$\Delta t(\mathrm{~s})$ & 10 & 10 & 60 & 80 & 10 \\
\hline$v_{j}(\mathrm{~m} / \mathrm{s})$ & 1.4 & 1 & 2 & 2 & 1 \\
\hline$Y_{j}$ & $135^{\circ}$ & $135^{\circ}$ & $0^{\circ}$ & $90^{\circ}$ & $45^{\circ}$ \\
\hline$x_{t}(\mathrm{~m})$ & 197 & 2 & 2 & 1 & 60 \\
\hline$x_{A}(m)$ & 139 & 142 & 2 & 141 & 2 \\
\hline$\overline{Y(m)}$ & 64 & 138 & 276 & 9 & 39 \\
\hline Current cell & 2 & 4 & 1 & 4 & 1 \\
\hline $\operatorname{Pr}\{H O A P 1\}(\%)$ & 0.83 & 0.62 & {$[70.06]$} & 99.90 & [100] \\
\hline $\operatorname{Pr}\{H O A P 2\}(\%)$ & [98.28] & 97.43 & 0 & 0.02 & 0 \\
\hline$\overline{P r\{H O A P 3\}}(\%)$ & 0.83 & 0.62 & 0 & 0.04 & 0 \\
\hline $\operatorname{Pr}\{H O A P 4\}(\%)$ & 0.06 & {$[1.32]$} & 29.94 & {$[0.04]$} & 0 \\
\hline
\end{tabular}
more than one waypoint during $\Delta t$. Since the pdf of the time between two waypoints is known [22,25], $\Delta t$ is found by solving

$$
\operatorname{Pr}\{\Delta t \leq T 1+T 2\} \leq \varepsilon,
$$

Table 2 Values and numerical results for the five cases analyzed with Maple

Values in square brackets stand for the probability of remaining in current cell. 
Table 3 Average percentages of $\mathrm{HO}$ and no $\mathrm{HO}$ for the five cases simulated with Matlab

\begin{tabular}{lccccc}
\hline Cases & $\mathbf{1}$ & $\mathbf{2}$ & $\mathbf{3}$ & $\mathbf{4}$ & $\mathbf{5}$ \\
\hline Pr\{HOAP1\} (\%) & 0.64 & 0.46 & {$[73.80]$} & 99.65 & {$[100]$} \\
\hline Pr\{HOAP2\} (\%) & {$[98.76]$} & 98.08 & 0.00 & 0.08 & 0.00 \\
\hline Pr\{HOAP3\} (\%) & 0.58 & 0.49 & 0.00 & 0.11 & 0.00 \\
\hline Pr\{HOAP4\} (\%) & 0.02 & {$[0.98]$} & 26.20 & {$[0.16]$} & 0.00 \\
\hline
\end{tabular}

Values in square brackets stand for the probability of remaining in current cell.

where $T 1$ and $T 2$ represent the timestamp (clock) of the first and of the second transitions, respectively. Table 1 shows the values for different probabilities of no more than one HO. In the cases presented below, this probability is set to a minimum of $95 \%$.

For the numerical cases, $R$ is set to $140 \mathrm{~m}$. The mobility pattern is RWP with a uniform speed distribution between 0.7 and $2 \mathrm{~m} / \mathrm{s}$ and a pause time of zero. The area is served by four APs as shown in Figure 1 (e.g., AP1 placed at point (70; 70) with respect to the centre of $A$ ). The cell range is $70 \sqrt{2} \mathrm{~m}$. After the equations in $[22,25]$, the mean distance between two waypoints is equal to $126.756 \mathrm{~m}$, the average time-weighted speed is $1.238 \mathrm{~m} / \mathrm{s}$ and the mean time between two waypoints is equal to $102.292 \mathrm{~s}$.

In order to present numerical results that illustrate some interesting cases, we selected five representative cases. The selected cases are far from representing all possible scenarios but are enough to support and facilitate a better understanding of the theoretical results. Table 2 shows the initial conditions for the cases under study. Polar coordinates are provided for $P_{t}$ and $\mathrm{WP}_{j}$. Both $x_{t}$ and $x_{A}$ are provided to show which calculation is performed (refer to Figure 4). The probability of $\mathrm{HO}$ to any cell is provided. Because the probability of $\mathrm{HO}$ to the current cell is obviously zero, the value provided in Table 2 corresponds to the probability of remaining (i.e., no $\mathrm{HO}$ ) and is enclosed in square brackets to avoid misunderstandings. The same notation is also used in Tables 3 and 4. The results are discussed after providing a better understanding of each case through Figure 9.

The five cases are depicted in Figure 9. The blue and red circles represent the maximum and minimum distances the node can walk if moving in any direction during $\Delta t$ at maximum and minimum speeds, respectively. The grey area represents all the possible points that the node can reach during $\Delta t$. The blue arrow represents the current direction of the movement. The current association to a cell is marked with darker boundaries. In cases 1 and 2, the node can take any direction and any speed without exiting $A$, while in the other cases there are directions and speeds that bring the node outside $A$ (i.e., it changes direction and speed before exiting $A$ ). The green star represents $P_{t}+v_{j} \cdot \Delta t$ (i.e., no waypoint is reached during $\Delta t$ )

In case 1 , the node has just entered cell 2 and it will $\mathrm{HO}$ only if it reaches a waypoint. As shown in Figure 9, if the node changes direction in $P_{t}$, it has roughly a $60 \%$ of chances to HO. While moving in the same direction, the probability of $\mathrm{HO}$ given that node changes direction will decrease (i.e., the grey crown shown in Figure 9 gets smaller as time elapses and its center moves toward the green star); this explains the low values for the probabilities of HO. Moreover, due to the symmetry of the layout, the probabilities of HO to AP1 and AP3 are the same, while the probability of $\mathrm{HO}$ to AP4 is very low.

In case 2, the node is approaching the border of cell 4 toward cell 2, so HO is highly probable. Only if the node reaches the waypoint before exiting cell 4 , it has some chances of remaining in the current cell or HO to AP1 or AP3. This explains the very low probabilities of $\mathrm{HO}$.

In case 3 , the node is reaching the boundaries of $A$, so the probability of reaching the waypoint during $\Delta t$ is

Table 4 Values for the other five cases analyzed with Maple and Matlab

\begin{tabular}{|c|c|c|c|c|c|}
\hline Cases & 6 & 7 & 8 & 9 & 10 \\
\hline$P_{t}$ & $(105 ; 0.74 \pi)$ & $(85 ; 0.26 \pi)$ & $(30 ; \pi / 2)$ & $(80 ; 3 \pi / 2)$ & $(30 ; \pi / 4)$ \\
\hline$W P_{j}$ & $(130 ; 0.72 \pi)$ & $(130 ; 0.22 \pi)$ & $(80 ; \pi / 4)$ & $(80 ; 5 \pi / 4)$ & $(20 ; 7 \pi / 4)$ \\
\hline$\Delta t(\mathrm{~s})$ & 60 & 80 & 80 & 50 & 80 \\
\hline$v_{j}(\mathrm{~m} / \mathrm{s})$ & 1.3 & 1.3 & 0.9 & 1.2 & 1.8 \\
\hline$\gamma_{j}$ & $295^{\circ}$ & $303^{\circ}$ & $205^{\circ}$ & $337^{\circ}$ & $79^{\circ}$ \\
\hline$x_{t}(\mathrm{~m})$ & 105.71 & 84.82 & 18.42 & 28.93 & 148.71 \\
\hline$x_{A}(m)$ & 235.80 & 216.55 & 150.09 & 88.29 & 114.05 \\
\hline$Y(\mathrm{~m})$ & 26.06 & 46.90 & 53.20 & 61.23 & 36.06 \\
\hline Current cell & 2 & 1 & 1 & 3 & 1 \\
\hline$\overline{P r}\{H O A P 1\}(\%)$ & $0.75 \mid 0.35$ & {$[20.77] \mid[22.02]$} & {$[3.78]||[2.73]$} & $0.02 \mid 0.01$ & {$[63.85] \mid[57.00]$} \\
\hline $\operatorname{Pr}\{H O A P 2\}(\%)$ & [98.46] | [99.21] & $68.20 \mid 70.76$ & 90.43 | 93.66 & $0.14 \mid 0.06$ & 19.59 | 25.30 \\
\hline $\operatorname{Pr}\{H O A P 3\}(\%)$ & $0.78 \mid 0.44$ & $2.04 \mid 0.96$ & $2.48 \mid 1.20$ & {$[18.66] \mid[15.06]$} & 5.35 | 3.21 \\
\hline $\operatorname{Pr}\{H O A P 4\}(\%)$ & $0.01 \mid 0.00$ & $8.99 \mid 6.27$ & $3.31 \mid 2.40$ & $81.18 \mid 84.87$ & $11.21 \mid 14.50$ \\
\hline
\end{tabular}

Values in square brackets stand for the probability of remaining in current cell. 


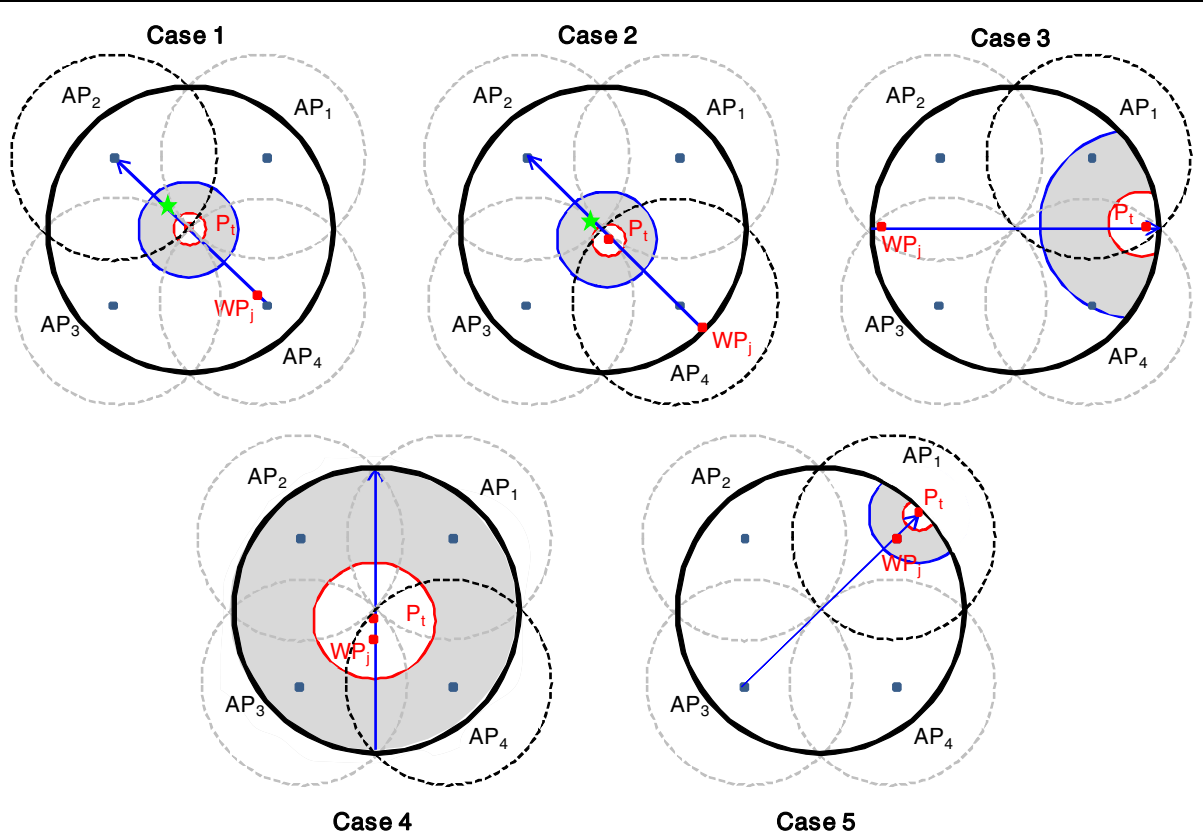

Figure 9 Area where the node can move if next waypoint is $\boldsymbol{P}_{\boldsymbol{t}}$ in each case. The minimum distance is represented in red, while the maximum is in blue. The current direction of movement is represented by the blue arrow. The boundaries of the AP to which the node is currently associated are highlighted. The green star represents $P_{t}+v_{j} \cdot \Delta t$ (i.e., no change of transition during $\Delta t$ ).

close to one. Since it is associated to cell 1 and the $\mathrm{HO}$ starts when the node leaves the cell, the percentage of HO to AP4 (29.94\%) is lower than the percentage of no $\mathrm{HO}-$ recall that it is shown inside square brackets in Table 2. Even at maximum speed, the node has no time to reach cell 2 or cell 3 , so the probability of $\mathrm{HO}$ to them is zero.

Case 4 is similar to case 2 . The percentage of $\mathrm{HO}$ is very high $(99.96 \%)$ since the node is approaching the border of its cell. Since the boundaries of cells 4 and 2 overlap at only one point (i.e., the center of $A$ ), the node will $\mathrm{HO}$ to AP1 with much higher probability. On the other hand, the probabilities of HO to AP2 and AP3 only accounts for the few chances that the node has to reach the waypoint before exiting its current cell.

Finally, case 5 is similar to case 3 . Again, the node will reach the waypoint because it is moving toward the boundaries of $A$. Because $\Delta t$ is short, the node has no opportunity to leave its cell, so the probability of $\mathrm{HO}$ is zero. This case differs from the others in that the node is not walking through the center of $A$.

\section{Simulation results}

The five cases analyzed are simulated with Matlab in order to evaluate the loss of accuracy produced by the necessary simplifications to the analytical model: numerical integration in (13), the approximation when the geometry is too complex, etc. Each simulation run consists of 5,000 samples and 10 independent repetitions for each run are performed for each case. This simulation length is long enough to guarantee a confidence interval better than $95 \%$ with a margin smaller than $10 \%$ in all cases. Table 3 shows the average percentages of $\mathrm{HO}-$ note that the value in square brackets represents the probability of remaining in the current cell.

All of the results from simulation match the analytical results. Due to the approximation in the analysis, slight differences may be noticed among the simulation and analytical results. Still, the error in the estimation of the probability of $\mathrm{HO}$ in the analysis with respect to that in the simulation is always lower than $4 \%$ (case 3). Thus, the simulation validates the analytical model.

For a more comprehensive validation, other five cases (see Figure 10) are analyzed both analytically and through simulation, and the results are presented in Table 4. The error in the probability of $\mathrm{HO}$ or no $\mathrm{HO}$ is always less than $7 \%$ (case 10). These results confirm that the proposed analytical framework is valid and that the approximations and simplifications followed have only a slight impact on the results.

\section{Conclusion}

An analytical model for the estimation of the probability of $\mathrm{HO}$ to a given cell of a node moving according to the RWP mobility model has been investigated. The method is presented for a layout in which the overall area is a circle with four APs located at the corners of a square inside it. It is assumed that the position and timestamp 


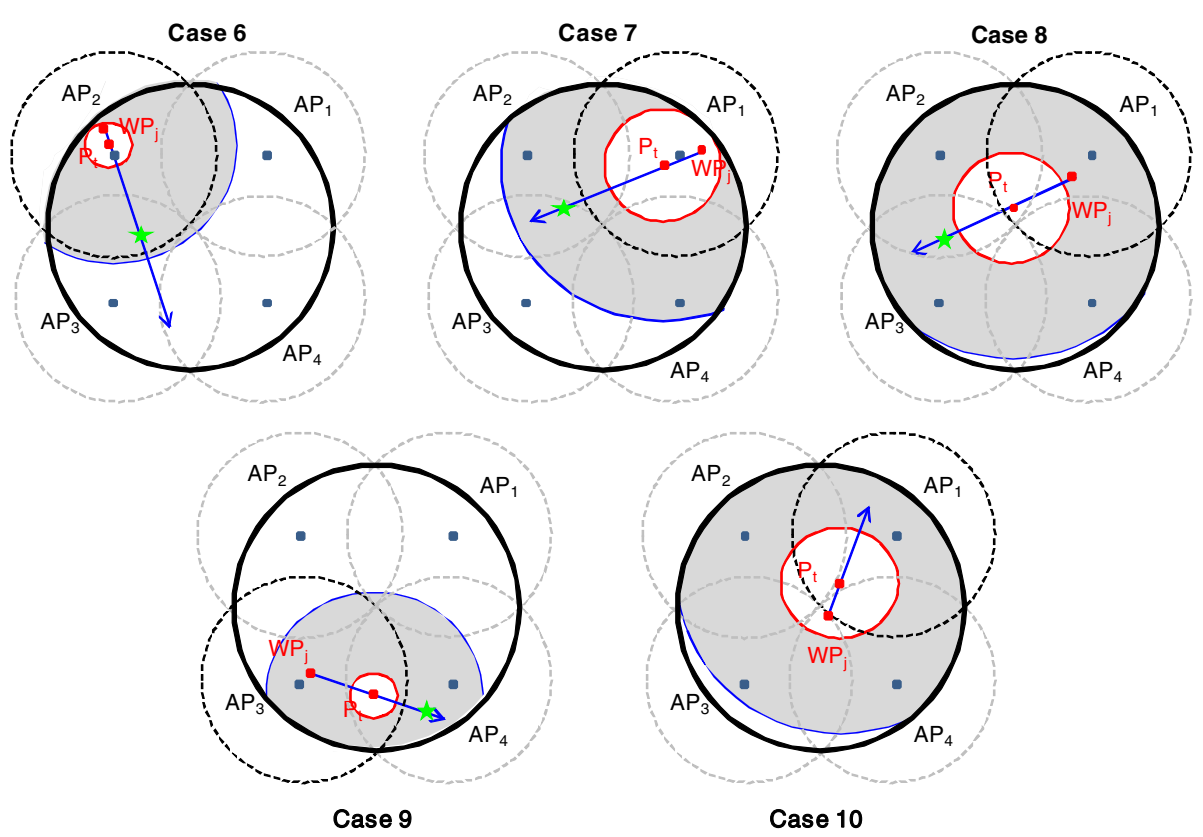

Figure 10 Area where the node can move if next waypoint is $P_{t}$ for each of the other five cases.

of the last waypoint are known. The model can be generalized to any symmetrical layout with overlapping areas, where the APs are placed at the corners of a regular polygon (i.e., triangle, hexagon). In this article, equations are provided for the case in which the speed distribution is uniform, the pause time is zero, and propagation conditions are ideal (i.e. only path loss). $\mathrm{Nu}$ merical results for several cases have been presented and compared with simulations to validate the approximations assumed in the mathematical analysis. This study provides the research community with an analytical framework which can improve the understanding and use of the RWP mobility model used in the majority of simulation studies with moving nodes. From a practical perspective, it can be used to improve resource allocation in cellular networks, thus smoothing the $\mathrm{HO}$ procedure and improving the QoS.

\section{Appendix 1}

Expressions for the distances used in the evaluation of the probability of $\mathrm{HO}$ in (13) are

$$
\begin{aligned}
& d 1(r)=\frac{1}{2}\left(\Delta t-\frac{r}{v_{j}}\right)^{2} \cdot\left(v_{\max }^{2}-v_{\min }^{2}\right), \\
& d 2(\theta, r)=\frac{1}{2}\left(\left(v_{\max } \cdot\left(\Delta t-\frac{r}{v_{j}}\right)\right)^{2}-x_{t}^{2}\left(\theta_{t}^{\prime}, r\right)\right),
\end{aligned}
$$

$$
d 3(\theta, r)=\frac{1}{2}\left(x_{A}^{2}(\theta, r)-\left(v_{\min } \cdot\left(\Delta t-\frac{r}{v_{j}}\right)\right)^{2}\right)
$$

According to the geometry, other distances may be used for the evaluation of $\operatorname{Pr}\{\mathrm{HOa}\}$

$$
\begin{aligned}
& d 4(\theta, r)=\frac{1}{2}\left(x_{A}^{2}(\theta, r)-x_{t}^{2}\left(\theta_{t}^{\prime}, r\right)\right) \\
& d 5(\theta, r)=\frac{1}{2}\left(x_{t A P}^{2}\left(\theta_{t}^{*}, r\right)-x_{t}^{2}\left(\theta_{t}^{\prime}, r\right)\right) \\
& d 6(\theta, r)=\frac{1}{2}\left(\left(v_{\max } \cdot\left(\Delta t-\frac{r}{v_{j}}\right)\right)^{2}-x_{t A P}^{2}\left(\theta_{t}^{*}, r\right)\right)
\end{aligned}
$$

Refer to Figure 6 for better understanding of these values.

\section{Appendix 2}

Generalization of Equations (2) to (4) in case the change of direction of movement and speed starts at $S(r)$ leads to the following equations

$$
\begin{aligned}
x_{A}\left(\theta_{t}(r), r\right)= & d_{t}(r) \cdot \cos \left(\theta_{t}(r)\right) \\
& +\sqrt{R^{2}-\left(d_{t}(r) \cdot \sin \left(\theta_{t}(r)\right)\right)^{2}},
\end{aligned}
$$




$$
\begin{aligned}
x_{t}\left(\theta_{t}^{\prime}(r), r\right)= & d^{\prime}{ }_{t}(r) \cdot \cos \left(\theta_{t}^{\prime}(r)\right) \\
+ & \sqrt{r_{\text {cell }}^{2}-\left(d_{t}^{\prime}(r) \cdot \sin \left(\theta_{t}^{\prime}(r)\right)\right)^{2}}, \\
x_{t A P \pm}\left(\theta_{t}^{*}(r), r\right)= & d^{*}{ }_{t}(r, A P) \cdot \cos \left(\theta_{t}^{*}(r)\right) \\
& \pm \sqrt{r_{\text {cell }}^{2}-\left(d^{*}{ }_{t}(r, A P) \cdot \sin \left(\theta_{t}^{*}(r)\right)\right)^{2}},
\end{aligned}
$$

where $d_{t}(r)$ is the distance of $S(r)$ to the center of $A, \theta_{t}$ $(r)$ is the new direction of movement, $d_{t}^{\prime}(r)$ is the distance of $S(r)$ to the center of the current cell, and $d_{t}^{*}(r$, $A P)$ is the distance of $S(r)$ to the border of the neighboring cell AP (as two intersections may occur, the symbol \pm stands for the two solutions). $\theta_{t}^{\prime}(r)$ and $\theta_{t}^{*}(r)$ are derived from (5) and (6), respectively. Refer to Figure 7 for a better understanding of these values.

\section{Competing interests}

The authors declare that they have no competing interests.

\section{Acknowledgments}

This research was supported by the Spanish Government and ERDF through CICYT projects TEC2009-08198. The authors would like to thank Dr. Javier Ozón Gorriz for his suggestions and valuable help with the mathematical analysis. Part of this study has been presented at IEEE LCN conference in October, 2011 [23].

Received: 5 June 2012 Accepted: 7 January 2013

Published: 25 January 2013

\section{References}

1. A Sgora, D Vergados, Handoff prioritization and decision schemes in wireless cellular networks: a survey. IEEE Commun. Surv. Tutor. 11(4), 57-77 (2009)

2. A Mishra, M Shin, W Arbaugh, An empirical analysis of the IEEE 802.11 MAC layer handoff process. SIGCOMM Comput. Commun. Rev. 33(2), 93-102 (2003)

3. H Velayos, G Karlsson, Techniques to reduce the IEEE $802.11 \mathrm{~b}$ handoff time, in Proceedings of the IEEE International Conference on Communications, vol.7, 2004, pp. 3844-3848

4. Y Iraqi, R Baoutaba, Handoff and call dropping probabilities in wireless cellular networks, in Proceedings of the IEEE International Conference on Wireless Networks, Communications and Mobile Computing, vol.1, 2005, pp. 209-213

5. WS Soh, HS Kim, QoS provisioning in cellular networks based on mobility prediction techniques. IEEE Commun. Mag. 41(1), 86-92 (2003)

6. DB Johnson, DA Maltz, Dynamic source routing in ad hoc wireless networks. Kluwer Academic Publishers Mob. Comput. 353, 153-181 (1996)

7. S Kurkowski, T Camp, M Colagrosso, MANET simulation studies: the incredibles. SIGMOBILE Mob. Comput. Commun. Rev. 9(4), 50-61 (2005)

8. T Ali, M Saquib, C Sengupta, Vertical handover analysis for voice over WLAN/ cellular network, in Proceedings of the IEEE International Conference on Communications, ed. by, 2010, pp. 1-5

9. C Tong, JW Niu, GZ Qu, X Long, XP Gao, Complex networks properties analysis for mobile ad hoc networks. IET Commun. 6(4), 370-380 (2012)

10. Q Min, R Zimmermann, An adaptive strategy for mobile ad hoc media streaming. IEEE Trans. Multimed. 12(4), 317-329 (2010)

11. A Jindal, K Psounis, Contention-aware performance analysis of mobility-assisted routing. IEEE Trans. Mob. Comput. 8(2), 145-161 (2009)

12. DN Alparslan, K Sohraby, Two-dimensional modeling and analysis of generalized random mobility models for wireless ad hoc networks. IEEE/ACM Trans. Netw. 15(3), 616-629 (2007)

13. K Tae-Hyong, Y Qiping, L Jae-Hyoung, P Soon-Gi, S Yeon-Seung, A mobility management technique with simple handover prediction for $3 \mathrm{G}$ LTE systems, in Proceedings of the IEEE 66th Vehicular Technology Conference, ed. by, 2007, pp. 259-263
14. A Rojas, P Branch, G Armitage, Validation of the random waypoint mobility model through a real world mobility trace, in Proceedings of the IEEE Region 10 TENCON, ed. by, 2005, pp. 1-6

15. T Camp, J Boleng, V Davies, A survey of mobility models for ad hoc network research. Wirel. Commun. Mob. Comput. (WCMC) (Special Issue on Mobile Ad Hoc Networking) 2(5), 483-502 (2002)

16. F Bai, N Sadagopan, A Helmy, IMPORTANT: a framework to systematically analyze the impact of mobility on performance of routing protocols for adhoc networks. in Proceedings of the Twenty-Second Annual Joint Conference of the IEEE Computer and Communications 2, 825-835 (2003)

17. TK Madsen, FHP Fitzek, R Prasad, Impact of different mobility models on connectivity probability of a wireless ad hoc network, in Proceedings of the International Workshop on Wireless Ad-Hoc Networks, ed. by, 2004, pp. $120-124$

18. P Lassila, E Hyytiä, H Koskinen, Connectivity properties of random waypoint mobility model for ad hoc networks, in Proceedings of the Fourth Annual Mediterranean Workshop on Ad Hoc Networks, ed. by, 2005, pp. 159-168

19. V Pla, V Casares-Giner, Analytical-numerical study of the handoff area sojourn time. in Proceedings of the IEEE Global Telecommunications Conference 1, 886-890 (2002)

20. E Hyytiä, J Virtamo, Random waypoint mobility model in cellular networks. ACM/Kluwer Wirel. Netw. 13(2), 177-188 (2007)

21. E Zola, F Barcelo-Arroyo, Impact of mobility models on the cell residence time in WLAN networks, in Proceedings of the IEEE Sarnoff Symposium, ed. by, 2009, pp. 1-5

22. C Bettstetter, H Hartenstein, X Pérez-Costa, Stochastic properties of the random waypoint mobility model. ACM/Kluwer Wirel. Netw. (Special Issue on Modeling and Analysis of Mobile Networks) 10(5), 555-567 (2004)

23. E Zola, F Barcelo-Arroyo, Probability of handoff for users moving with the random waypoint mobility model, in Proceedings of the IEEE LCN, 2011, pp. 187-190

24. C Bettstetter, G Resta, P Santi, The node distribution of the random waypoint mobility model for wireless ad hoc networks. IEEE Trans. Mob. Comput. 2(3), 257-269 (2003)

25. E Hyttiä, P Lassila, J Virtamo, Spatial node distribution of the random waypoint mobility model with applications. IEEE Trans. Mob. Comput. 5(6), 680-694 (2006)

26. W Yueh-Ting, H Tsung-Yen, L Wanjiun, T Cheng-Lin, Epoch, length of the random waypoint model in mobile ad hoc networks. IEEE Commun. Lett. 9(11), 1003-1005 (2005)

27. S Sangho, AG Forte, R Anshuman Singh, H Schulzrinne, Reducing MAC layer handoff latency, in IEEE 802.11 wireless LANs, in Proceedings of the ACM Second International Workshop on Mobility Management \& Wireless Access Protocols, 2004, pp. 19-26

28. K Ramachandran, S Rangarajan, JC Lin, Make-before-break MAC layer handoff in 802.11 wireless networks, in Proceedings of the IEEE ICC, vol.10, 2006, pp. 4818-4823

29. L Yong, G Lixin, Practical schemes for smooth MAC layer handoff in 802.11 wireless networks, in Proceedings of the IEEE Computer Society International Symposium on World of Wireless, Mobile and Multimedia Networks, 2006, pp. 181-190

30. J Márquez-Barja, C Calafate, JC Cano, P Manzoni, An overview of vertical handover techniques: algorithms, protocols and tools. Elsevier. Comput. Commun. 34(8), 985-997 (2011)

31. X Yan, YA Şekercioğlu, S Narayanan, A survey of vertical handover decision algorithms in Fourth Generation heterogeneous wireless networks. Elsevier Comput. Netw. 54(11), 1848-1863 (2010)

32. S Lee, K Kim, K Hong, D Griffith, YH Kim, N Golmie, A probabilistic call admission control algorithm for WLAN in heterogeneous wireless environment. IEEE Trans. Wirel. Commun. 8(4), 1672-1676 (2009)

33. R Ben Ali, S Pierre, On the impact of soft vertical handoff on optimal voice admission control in PCF-based WLANs loosely coupled to $3 \mathrm{G}$ networks. IEEE Trans. Wirel. Commun. 8(3), 1356-1365 (2009)

34. PN Pathirana, AV Savkin, S Jha, Mobility modelling and trajectory prediction for cellular networks with mobile base stations, in Proceedings of the 4th ACM International Symposium on Mobile Ad Hoc Networking \& Computing, 2003,pp. 213-221

35. T Joshi, A Mukherjee, DP Agrawal, Exploiting mobility patterns to reduce reauthentication overheads in infrastructure WLAN networks, in Proceedings of the IEEE Canadian Conference on Electrical and Computer Engineering, 2006, pp. 1423-1426 
36. S Michaelis, C Wietfeld, Comparison of user mobility pattern prediction algorithms to increase handover trigger accuracy, in Proceedings of the IEEE Vehicular Technology Conference, vol.2, 2006, pp. 952-956

37. MS Sricharan, $V$ Vaidehi, A pragmatic analysis of user mobility patterns in macrocellular wireless networks. Elsevier Pervas. Mob. Comput. 4(5), 616-632 (2008)

38. PS Prasad, P Agrawal, Movement prediction in wireless networks using mobility traces, in Proceedings of the 7th IEEE Conference on Consumer Communications and Networking Conference, 2010, pp. 1-5

39. S Libo, D Kotz, J Ravi, H Xiaoning, Evaluating next-cell predictors with extensive wi-fi mobility data. IEEE Trans. Mob. Comput. 5(12), 1633-1649 (2006)

40. W Navidi, T Camp, Stationary distributions for the random waypoint mobility model. IEEE Trans. Mob. Comput. 3(1), 99-108 (2004)

doi:10.1186/1687-1499-2013-16

Cite this article as: Zola et al.: Forecasting the next handoff for users

moving with the Random Waypoint mobility model. EURASIP Journal on Wireless Communications and Networking 2013 2013:16.

\section{Submit your manuscript to a SpringerOpen ${ }^{\circ}$} journal and benefit from:

- Convenient online submission

- Rigorous peer review

- Immediate publication on acceptance

- Open access: articles freely available online

- High visibility within the field

- Retaining the copyright to your article 